\title{
La Modernidad más austral de Chile: el edificio de la U.T.E. en Punta Arenas (1964-1968), un ilustre desconocido.
}

\author{
Max Aguirre González - Juan José San Martín Pincheira
}

\section{Resumen}

La obra de la Universidad Técnica del Estado (1964-1968) en Punta Arenas (latitud $53^{\circ} 8^{\prime} 53,3^{\prime \prime}$ sur, longitud $70^{\circ} 55^{\prime} 41,72^{\prime \prime}$ oeste) jalona una etapa de la modernidad local y pone en evidencia la ambigüedad del término moderno, que obliga a la precisión de la categoría cada vez que circunscribimos el estudio a un caso específico como éste.

\section{Palabras Claves:}

MOVIMIENTO MODERNO. PUNTA ARENAS. UNIVERSIDAD TÉCNICA DEL ESTADO. ARQTOS. BRESCIANI, VALDÉS, CASTILLO Y HUIDOBRO.

\section{La iniciativa de la obra en su contexto}

En la ciudad de Punta Arenas (Fig.1), en el período 1953-1964, debido a "la insuficiencia, antigüedad e incomodidad de muchos locales"1, hubo un auge de nuevos establecimientos educacionales. En enero de 1961, Jorge Cvitanic, regidor de la Municipalidad de Magallanes, “propuso a la corporación que se solicitara a la Universidad Técnica del Estado, de Santiago, la creación de cursos técnicos de nivel superior a fin de ofrecer una alternativa de educación universitaria a los jóvenes magallánicos" ${ }^{2}$, con la intención de poder contar con profesionales que contribuyeran al desarrollo industrial de la región y, además, "conjurar" la amenaza de desarraigo a la que se veían sometidos los estudiantes que debían emigrar para realizar sus estudios universitarios.

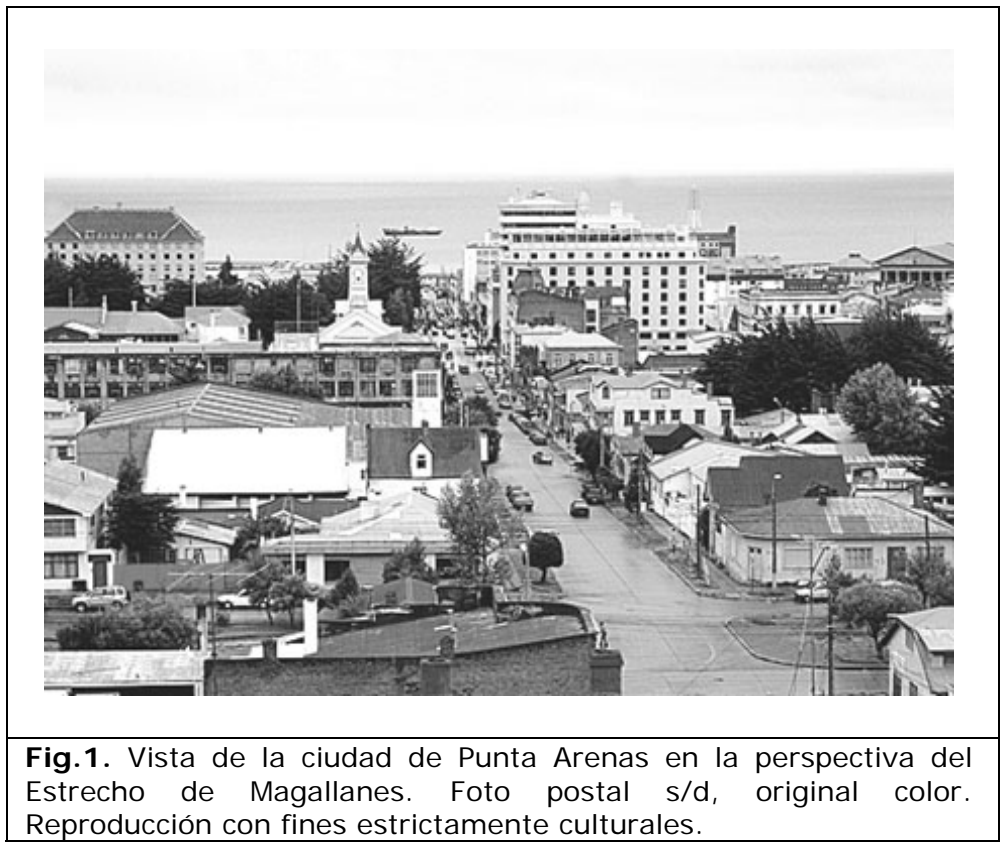




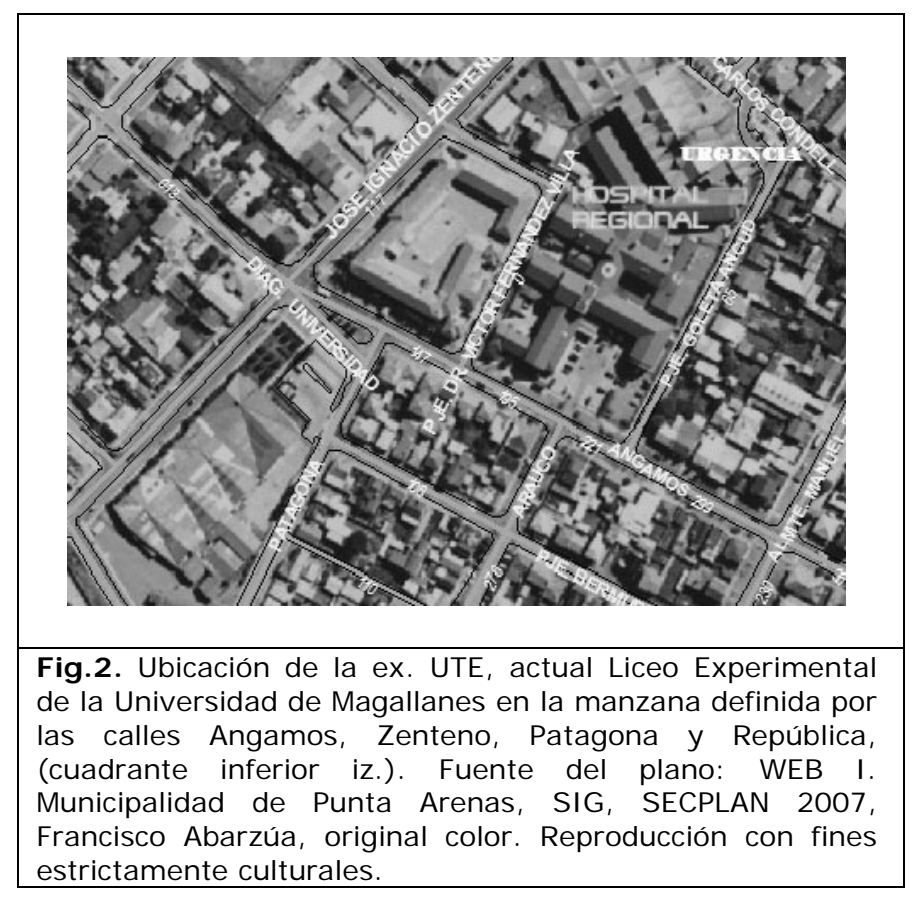

La iniciativa tuvo rápida acogida y en mayo de 1961 se inauguraron cursos de electricidad y mecánica industriales dictados por la Universidad en Punta Arenas ${ }^{3}$. Con posterioridad, durante el gobierno de Frei Montalva, a través del gobierno regional, se continuó prestando apoyo a los cursos desarrollados por la U.T.E., los que en 1965 y 1968 fueron ampliados a las especialidades de petroquímica, contabilidad pública y enfermería. En 1967 la matrícula de 1964 ya había sido superada tres veces, alcanzando a tener 348 estudiantes. En este contexto, caracterizado por la aceptación de la comunidad y la confianza en el futuro de la actividad universitaria, se creó la sede de Punta Arenas de la Universidad Técnica del Estado 4 .

La oficina de los arquitectos Bresciani, Valdés, Castillo y Huidobro ${ }^{5}$ ejecutaba las obras de la Unidad Habitacional Villa Portales (1954-1964), en el sector de Quinta Normal de Santiago, aledaño a los terrenos de la Universidad Técnica del Estado, cuando el Rector de esa Universidad tomó contacto con ellos (1957) iniciando una relación profesional que comprendió la realización de las sedes de Santiago (1957-1962), Antofagasta (1959), Concepción (1960) y Punta Arenas (1963).

La sede de la Universidad Técnica del Estado en Punta Arenas, corresponde, así, al esfuerzo que llevó adelante esa Universidad para crear una red nacional de instituciones de enseñanza superior, que abordarían la formación técnica en la década de 1960. Fue un plan de política de desarrollo a través de la formación de cuadros profesionales en áreas de alto impacto en la producción industrial ${ }^{6}$. A partir de la necesidad de formar técnicos capacitados, los gobiernos radicales impulsaron en los años treinta la educación técnica superior en el país: Aguirre Cerda, Ríos y González Videla lideraron el proceso educativo superior creando en 1947 la Escuela de Artes y Oficios la que en 1961 pasó a ser la Universidad Técnica del Estado (U.T.E.) expandiéndose desde Arica hasta Punta Arenas. 


\section{Hechos en torno al proyecto}

Siendo Intendente Mateo Martinic Beros, durante 1964, se desarrolló el proyecto de la sede en un periodo de tres meses (abril- junio) en un conjunto de 30 planos $^{7}$, en una época que se llevan a cabo numerosas obras públicas ${ }^{8}$.

En 1966 aún se discutía la aprobación de la ley que destinaría fondos para la construcción de la sede universitaria que, por un momento, un veto presidencial frenó. Las reacciones no se hicieron esperar, representantes de diversas organizaciones dieron a conocer su preocupación públicamente enviando solicitudes de reconsideración, especialmente, a los parlamentarios regionales. Entre ellos, el presidente del Centro para el Progreso, el Alcalde, la Cámara de Comercio e Industrias de Magallanes, el Club de Leones, el Consejo Provincial de la Central Única de Trabajadores, periodistas ${ }^{9}$.

A los pocos días de haber sido noticia el veto presidencial La Prensa Austral informaba la superación del impasse señalando que habían sido autorizados fondos para proseguir las obras que ya se habían comenzado ${ }^{10}$. Los antecedentes expuestos ponen en evidencia que las obras no siempre revelan esos aspectos que exceden a las consideraciones exclusivas de la arquitectura, pero que al ponerlos como telón de fondo en la perspectiva del interés patrimonial, confieren a la obra nuevas luces que aumentan su valoración.

El 18 de abril de 1968 el Ministro de Obras Públicas, Sergio Ossa Pretot, en visita a las obras señaló: "el Fisco ha realizado un esfuerzo gigante, con una inversión de 5.700.000 escudos. Como proyecto arquitectónico es hermoso y audaz. Hay plena disposición de las salas. La UTE magallánica tendrá el primer laboratorio de petroquímica de Chile"11.

Nueve días después se inauguró en presencia del Presidente de la República Eduardo Frei Montalvo, y el Ministro Ossa Pretot, completó la impresión sobre el edificio diciendo:

“(...) El edificio de la Universidad Técnica del Estado que ahora inauguramos responde a una moderna planificación escolar. El proyecto fue elaborado por los distinguidos arquitectos, señores Carlos Bresciani, Héctor Valdés, Fernando Castillo y Carlos Huidobro, que actualmente ocupan destacadas funciones en la vida universitaria y administrativa nacional y ejecutado con enorme interés y cariño por la firma Dieter Meyer. Vayan para todos los agradecimientos del Gobierno de Chile" ${ }^{12}$.

\section{La obra}

La obra se organiza en torno a un eje que la cruza longitudinalmente, partiendo perpendicularmente desde el volumen de acceso principal. En torno a él se disponen a un lado las naves de los grandes talleres y al otro los recintos de servicios de menor espacialidad (Fig.3). 


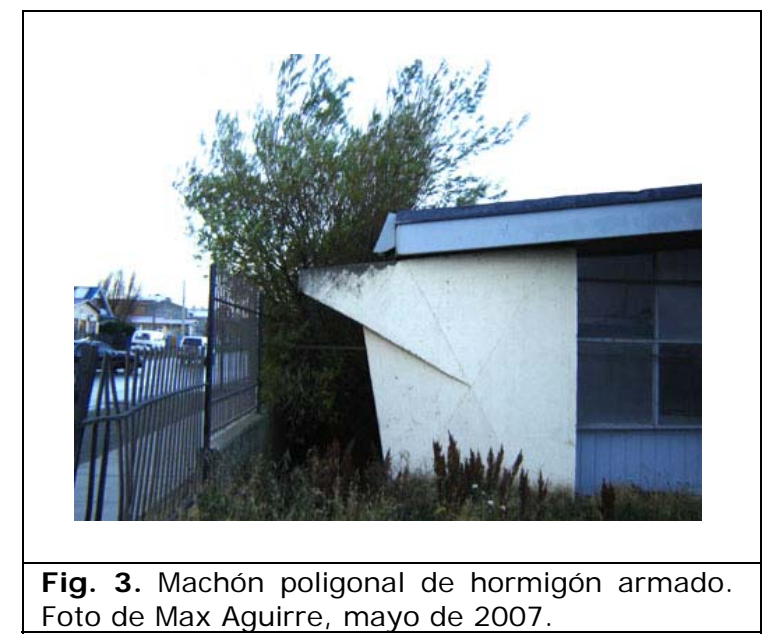

El total queda contenido en un perímetro rectangular con plantas en distintos niveles, en algunos casos de doble altura. El resultado es un conjunto con una distribución clara y concentrada que daba cabida a 400 alumnos en $4.140 \mathrm{~m}^{2}$ repartidos entre administración, docencia, laboratorios y esparcimiento. Los recursos financieros fueron otorgados por la Ley 14.824 que proveyó de CHILE-E 5.000 .000 (cinco millones de Escudos) invertidos entre 1965 y 1968.

Con gran acierto, el Ministro Ossa Pretot describió el edificio el día de la inauguración diciendo "La obra es una unidad compacta como respuesta al medio climático de Magallanes y con un claro sentido de integración docente"13. El espacio de talleres caracteriza el edificio por el uso de vigas metálicas de gran luz, que arrancan de unas piezas poligonales aisladas de hormigón armado, que se observan en los lados del perímetro de mayor longitud como nuevos atlantes (Fig. 4).

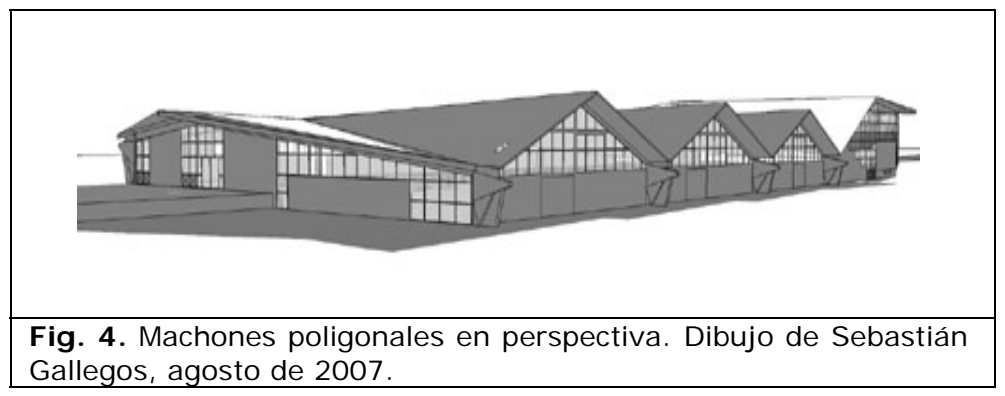

El efecto exterior, en perspectiva aérea, es una gran cubierta de planos triangulares desplegados, donde limahoyas y limatesas articulan los quiebres de los planos, acentuando la unidad del conjunto (Fig. 5). Héctor Valdés afirmó que "la idea fue hacer un gran paraguas, considerando el clima y, el acero, fue por la rapidez"14.

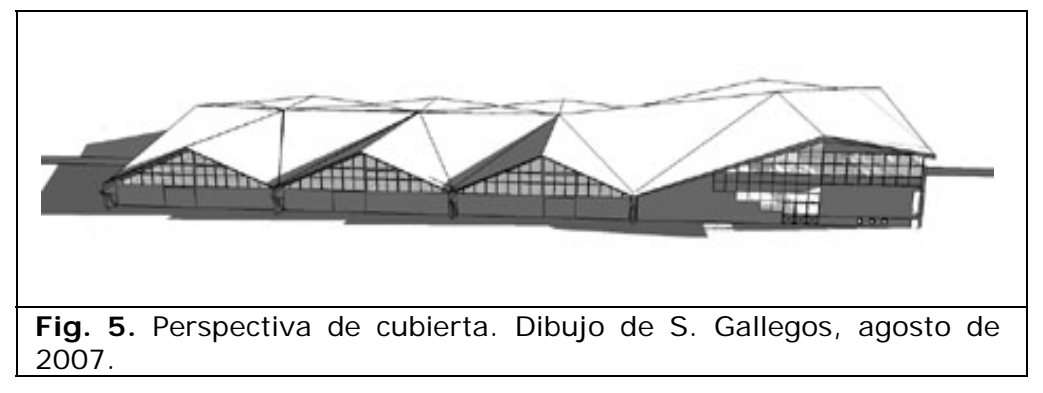


La obra introdujo en la ciudad el uso racional en la estructura de los nuevos materiales, destacable son los machones poligonales de hormigón armado (Fig. 6) que sostienen las vigas metálicas. Desde ellos se alzan las vigas desde una altura no mayor de 2,5 m elevándose en línea recta hasta alcanzar la altura máxima de los talleres.

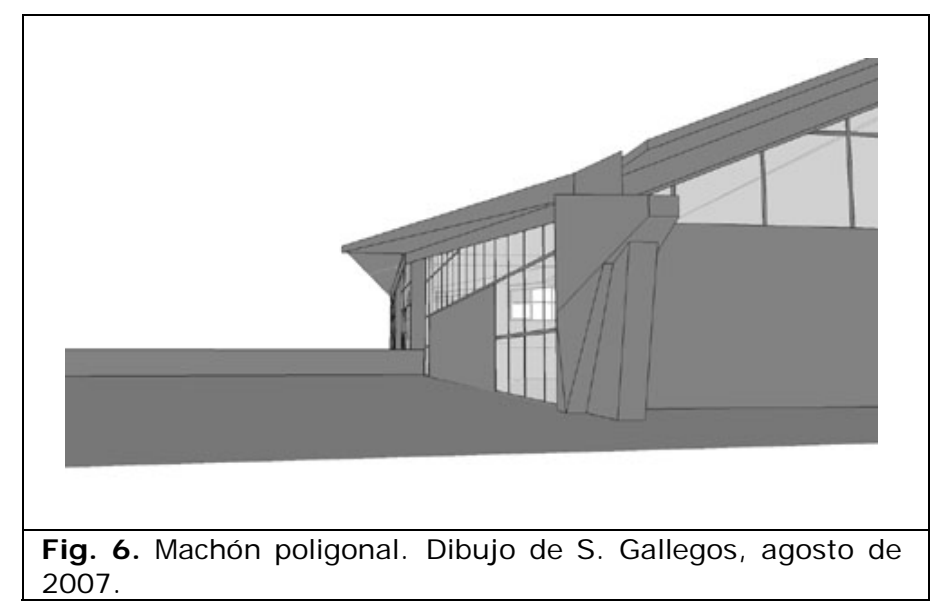

La organización compacta en torno al eje de circulación satisface los requerimientos de la enseñanza integral que se promovía en la época y favorece el enfrentamiento del rigor climático, que se completa con la creación de un patio cubierto de gran superficie para actividades recreativas. El resultado es una obra de gran sencillez formal, de geometría abstracta, que aborda con claridad espacial y de orden la solución del centro de enseñanza técnica superior (Figs.7-8).
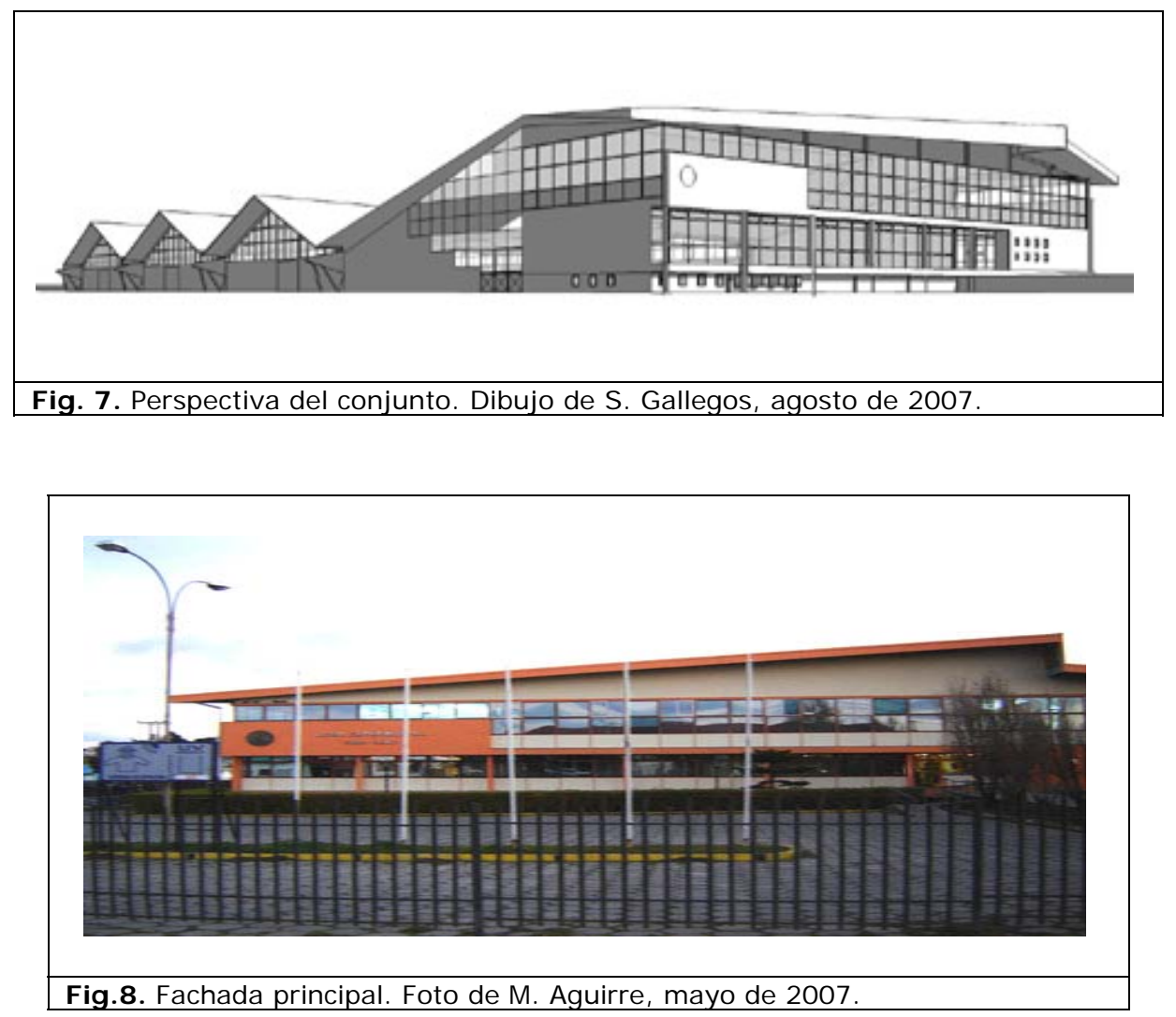


\section{La obra y su impacto frente a la arquitectura de la ciudad de Punta Arenas}

Más allá de estas consideraciones descriptivas, la obra puso a la ciudad en sintonía con el estado del discurso moderno en el país, en circunstancias que la arquitectura de actualidad en Punta Arenas fluctuaba entre experiencias rezagadas de la modernidad común en el país en los años cuarenta, caracterizada por la ausencia de elementos históricos, volúmenes macizos de líneas rectas y algún detalle de borde o cornisa que hacía guiños al Art Decó (el caso de la ex-Oficina de Tierras en calle Bories casi esquina de José Menéndez) (Fig.9), y la arquitectura de tipologías estándares que venía imponiendo la S.C.E.E. ${ }^{15}$ desde 1937 en los establecimientos educacionales (los casos de las Escuelas 14, de Barranco Amarillo (Fig. 10) y 12, de la Población Williams (Fig.11).

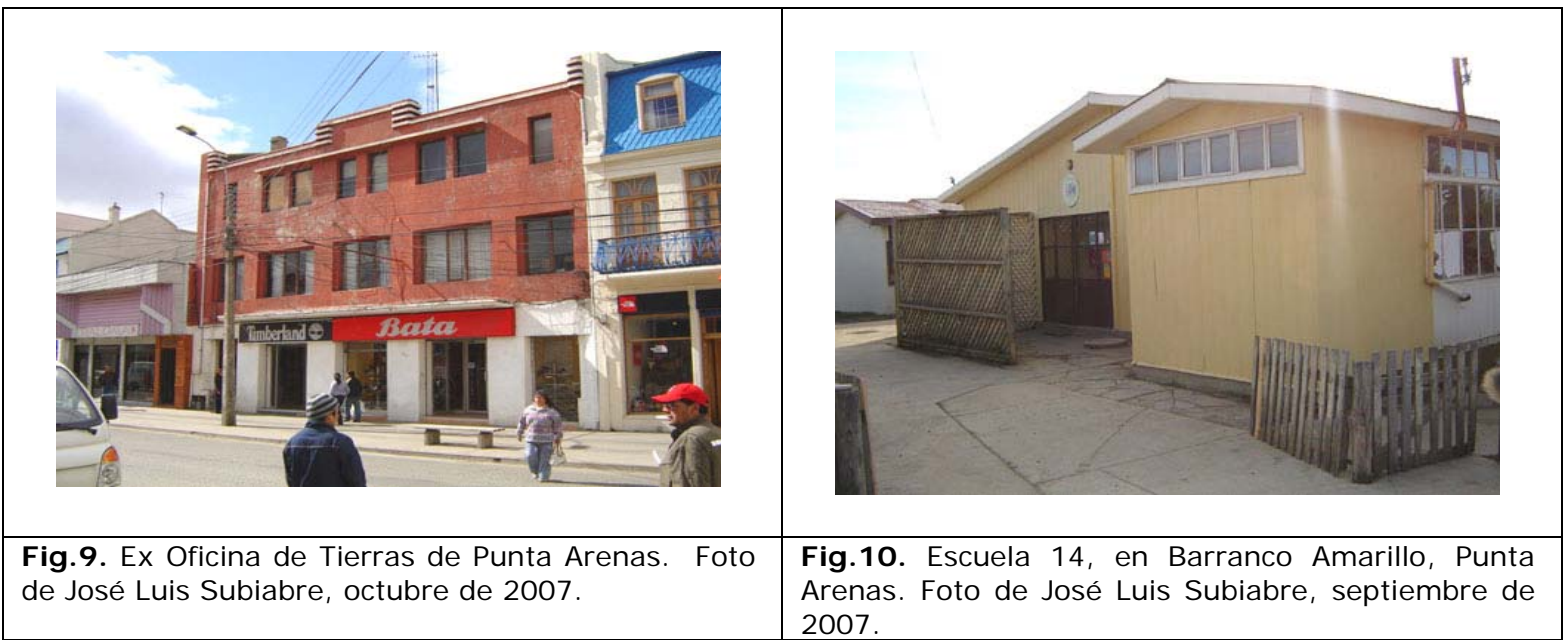

de José Luis Subiabre, octubre de 2007. Arenas. Foto de José Luis Subiabre, septiembre de 2007.

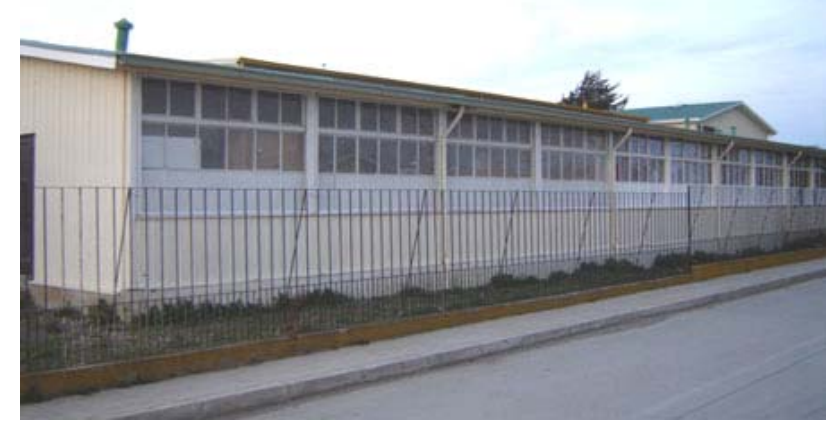

Fig. 11. Escuela 12 de la Población Williams, Punta Arenas. Foto de José Luis Subiabre, octubre de 2007.

En este sentido la obra de la U.T.E. en Punta Arenas jalona una etapa de la modernidad local y pone en evidencia (es un caso más en la historia de la modernidad arquitectónica en el país) la ambigüedad del término moderno, que obliga a la precisión de la categoría cada vez que circunscribimos el estudio a un caso específico como éste (Fig.12). 


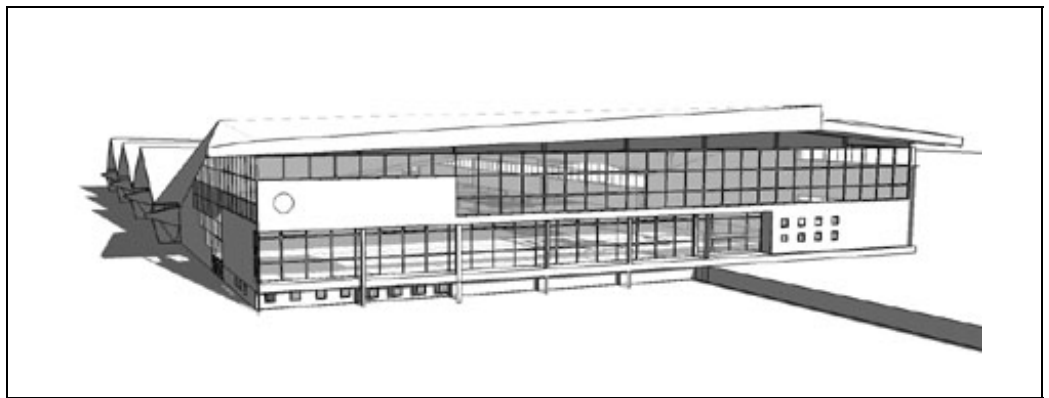

Fig. 12. Perspectiva del conjunto. Dibujo de S. Gallegos, agosto de 2007.

Finalmente, cabe denunciar el deterioro que se ha producido a través del tiempo. La obra se fue rodeando de pequeñas "ampliaciones" de esa común factura de "lo provisorio permanente", que dentro de todo son inofensivas. Pero, este año ha sido seriamente dañada con una intervención inmisericorde, que introdujo en medio de los talleres una estructura del tipo galpón industrial estándar (Fig.13), demoliendo al menos uno de los machones con las vigas asociadas a él. Bajo tales circunstancias, estos trabajos de divulgación, balancean su significado entre la carta de presentación y el obituario de un ilustre desconocido.

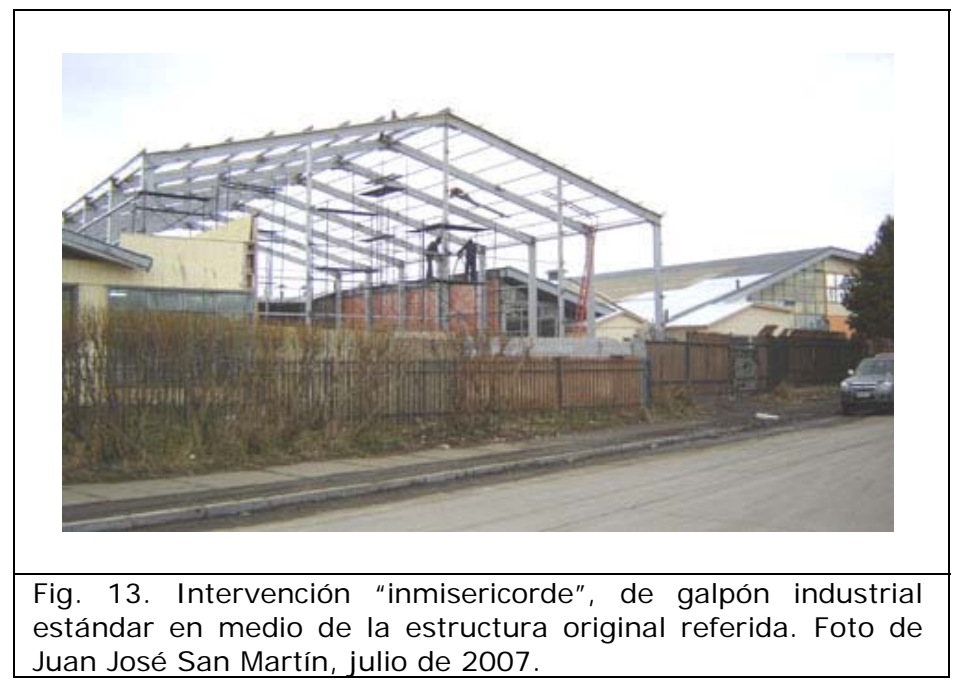

N. de R.:

Max Aguirre G. es Dr. Arquitecto, Académico del Departamento de Historia y Teoría de la Arquitectura en la Facultad de Arquitectura y Urbanismo de la Universidad de Chile.

maxaguirre@uchile.cl

Juan José San Martín P. es Arquitecto, Profesor en la Escuela de Arquitectura de la Universidad de Magallanes. Colaboró en la investigación Mariel Rubio Araya, Licenciada en Historia (PUC); y la modelación 3D fue realizada por Sebastián Gallegos Trujillo, estudiante de arquitectura de la Universidad de Magallanes. juan.sanmartin@umag.cl 
${ }^{1}$ MARTINIC, Mateo. Historia de la región magallánica, Vol. II, Punta Arenas, Universidad de Magallanes, 1992, p1213.

2 Op. cit., p1216.

3 La Prensa Austral, 27 de abril, 1968, en “Oficio N 546 de 21 de abril de 1961, (...) la Ilustre Municipalidad comunica que ha llegado a un acuerdo con la Confederación Deportiva de esta ciudad para ocupar las salas del Gimnasio Cubierto, las que reúnen las condiciones necesarias para el funcionamiento de los cursos creados por la Universidad Técnica del Estado".

4 Ubicada en la manzana comprendida entre las calles Angamos, Zenteno, Patagona y República, actualmente funciona allí el Liceo Experimental de la Universidad de Magallanes.

5 La Oficina Bresciani, Valdés, Castillo y Huidobro, fue una de las más importantes que tuvo el país durante el siglo XX, constituida por los Arquitectos Carlos Bresciani Bagattini, Héctor Valdés Philips, Fernando Castillo Velasco y Carlos García Huidobro. Su extensa realización abarca obras de envergadura desde Arica a Punta Arenas. El Arquitecto Carlos Bresciani, recibió en forma póstuma el Premio Nacional de Arquitectura, en 1970; en 1976 lo recibió Héctor Valdés y Fernando Castillo Velasco en 1983. Esta Oficina se formó en1944.

6 La Prensa Austral, 27 de abril, 1968. En 1968, la institución contaba con 8 sedes de Provincia y Santiago, 31 carreras universitarias y una matrícula de 17.000 alumnos, desde Antofagasta a Punta Arenas.

7 Fondo Bresciani, Valdés, Castillo, Huidobro, Archivo de Originales, Centro de Información y Documentación Sergio Larraín García-Moreno, Pontificia Universidad Católica de Chile, Escuela de Arquitectura.

${ }^{8}$ Carlos Descouvieres Gómez, arquitecto provincial, afirmó que había fondos para diversas obras públicas en la región, por ejemplo, para el nuevo edificio de la Oficina de Tierras, ubicado en calle Bories casi esquina Valdivia (actual calle José Menéndez), el Retén fronterizo en Gallegos Chico y la Hostería del Paine; La Prensa Austral, viernes 11 de diciembre, 1964. Además, durante el año de la inauguración de la sede universitaria, están en ejecución o en vías de realización el Gimnasio del Liceo de Hombres de Punta Arenas, el Gimnasio cubierto de Porvenir, el Estadio de Punta Arenas, las Escuelas 14 de Barranco Amarillo y 12 de la Población Williams, más las ampliaciones de la Escuela 1 de Puerto Porvenir, en Tierra del Fuego y 6 de Puerto Natales en Ultima Esperanza y, se está terminando el muelle de Punta Arenas; La Prensa Austral, domingo 28 de abril, 1968.

${ }^{9}$ El senador por la circunscripción Julio Von Muhlembrock dirigió al director de la Universidad Técnica, Roberto Bravo, y a los órganos de difusión regionales, el siguiente telegrama: "Siento comunicarle que el Presidente de la República vetó la disposición de la ley de Presupuesto que disponía invertir la totalidad de fondos Ley Magallanes para la construcción de la Universidad Técnica en Punta Arenas (...)". El alcalde Carlos González Jaksic, hizo lo suyo enviando una comunicación a los parlamentarios regionales. El Consejo Provincial de la Central Única de Trabajadores, despachó esta comunicación: “Consternación ha causado veto del Ejecutivo utilización de fondos Ley Puerto Libre Construcción UTE Parenas [sic] (...)". El telegrama de la CUT fue dirigido a los senadores Aniceto Rodríguez, Carlos Contreras, Sergio Sepúlveda, Ezequiel González y Julio Von Muhlembrock, y al diputado Ernesto Guajardo. Los periodistas se hicieron presente, mediante la siguiente nota telegráfica enviada al presidente de la Comisión de Hacienda del Senado: "Circulo de la Prensa, interpretando el anhelo especialmente de los sectores populares por adquirir conocimientos técnicos profesionales, lamenta veto presidencial partida destinada a la construcción de la Universidad Técnica del Estado. Rogamos posible reconsideración de este veto que obstruye expectativas especialmente a sectores modestos". Firmaron esta declaración José Kramarenko, presidente y Francisco Eterovic, pro- secretario; La Prensa Austral, 7 de enero, 1966.

10 "En la intendencia de la Provincia se nos informó que la construcción de la Escuela Universitaria de Punta Arenas, de la Universidad Técnica del Estado, cuenta con financiamiento asegurado para el año 1966, ya que se dispone de la suma de $E^{\circ} 500.000$, suma que está asegurada y que alcanza para proseguir normalmente los trabajos que ya se han empezado. Se nos dijo que el veto presidencial al financiamiento tuvo su origen en que la moción parlamentaria correspondiente pretendía utilizar la totalidad de los fondos de la ley 14.284, dejando de esta manera sin financiamiento a otras obras en actual ejecución, tales como la pavimentación urbana en Punta Arenas, Puerto Natales y Porvenir, 
pavimentación del muelle Arturo Prat, obras diversas que ejecuta el Departamento de Arquitectura, etc. La Intendencia señaló que la ley 14.824 fue concebida para "suplementar" obras públicas con fondos producidos en Magallanes. En ningún caso, se nos dijo, es para financiar totalmente una obra, como se ha pretendido en la iniciativa parlamentaria que mereció el veto del Ejecutivo"; La Prensa Austral, 11 de enero, 1966.

${ }^{11}$ La Prensa Austral, 19 de abril, 1968.

${ }^{12}$ La Prensa Austral, 27 de abril, 1968. En la misma ocasión el Secretario General de la Universidad Técnica del Estado, Héctor Torres Guerra, se refirió así en el discurso inaugural "Con este moderno edificio nada tiene que envidiar esta esforzada zona a los que nuestra Universidad posee en Santiago y otras ciudades del país. Se incorpora así esta sede, al Plan de Construcciones que ha proyectado la Superioridad y ello gracias a la decisión, a la generosidad y al esfuerzo de toda la ciudad de Punta Arenas y la provincia de Magallanes".

13 La Prensa Austral, 27 de abril, 1968.

${ }^{14}$ Conversación con el arquitecto Héctor Valdés P., martes 14 de agosto de 2007.

${ }^{15}$ Sociedad Constructora de Establecimientos Educacionales (1937-1987). 